This is a pre-copyedited version of a contribution published in T. Ahram, W. Karwowski, A. Vergnano, F. Leali, \& R. Taiar (Eds.), Intelligent Human Systems Integration 2020 (pp. 932-937) published by Springer International Publishing. The definitive authenticated version is available online via https://doi.org/10.1007/978-3-030-395124_142. Cite as:

Beggiato, M., Rauh, N., \& Krems, J. (2020). Facial Expressions as Indicator for Discomfort in Automated Driving. In T. Ahram, W. Karwowski, A. Vergnano, F. Leali, \& R. Taiar (Eds.), Intelligent Human Systems Integration 2020 (pp. 932-937). Cham: Springer International Publishing. doi:10.1007/978-3-030-39512-4_142.

\title{
Facial Expressions as Indicator for Discomfort in Automated Driving
}

\author{
Matthias Beggiato ${ }^{1}$, Nadine Rauh ${ }^{1}$ and Josef Krems ${ }^{1}$ \\ ${ }^{1}$ Chemnitz University of Technology, Cognitive and Engineering Psychology, \\ Wilhelm-Raabe-Str. 43, 09120 Chemnitz, Germany \\ \{matthias.beggiato, nadine.rauh, josef.krems\}@psychologie.tu-chemnitz.de
}

\begin{abstract}
Driving comfort is considered a key factor for broad public acceptance of automated driving. Based on continuous driver/passenger monitoring, potential discomfort could be avoided by adapting automation features such as the driving style. The EU-project MEDIATOR (mediatorproject.eu) aims at developing a mediating system in automated vehicles by constantly evaluating the performance of driver and automation. As facial expressions could be an indicator of discomfort, a driving simulator study has been carried out to investigate this relationship. A total of 41 participants experienced three potentially uncomfortable automated approach situations to a truck driving ahead. The face video of four cameras was analyzed with the Visage facial feature detection and face analysis software, extracting 23 Action Units (AUs). Situation-specific effects showed that the eyes were kept open and eye blinks were reduced (AU43). Inner brows (AU1) as well as upper lids (AU5) raised, indicating surprise. Lips were pressed (AU24) and stretched (AU20) as sign for tension. Overall, facial expression analysis could contribute to detect discomfort in automated driving.
\end{abstract}

Keywords: face tracking · facial expressions · action units · automated driving · discomfort $\cdot$ driving simulator $\cdot$ mediator project

\section{Introduction}

Comfort is considered as one of the main drivers for higher levels of automated driving next to safety, efficiency, social inclusion and accessibility [1]. Although there is no common definition of comfort, it can be considered a feeling of well-being and an attribution of positive valence, associated with the absence of discomfort and uneasiness [2]. Next to traditional comfort aspects such as vibrations, noise or sitting comfort, new and additional determinants are discussed in automated driving such as apparent safety, trust in the system, feeling of control, motion sickness, familiarity of driving maneuvers as well as information about system states and actions [3, 4]. As these new comfort aspects are mainly related to specific and dynamic situations, constant evaluation is required. Based on this continuous driver/passenger comfort evaluation, potential discomfort could be avoided by adapting automation features. The basic idea of such comfort-adaptive automation is the metaphor of a vehicle-driver-team that knows each other's strengths, limitations, and current states and can react accordingly. 
The EU-project MEDIATOR (mediatorproject.eu) aims at developing a mediating system in automated vehicles, pursuing a paradigm shift away from a view that prioritizes either the driver or the automation, instead integrating the best of both. The mediating system should intelligently assess the strengths and weaknesses of both the driver and the automation and mediate between them, while simultaneously taking into account the driving context. As part of the driver/passenger state, facial expressions could be one indicator of potential discomfort during automated driving. If detected accordingly, automation features such as driving style aspects (e.g. speed, lateral distance, distance to vehicle ahead) or information presentation could be adapted to prevent uncomfortable situations, disengagement of automation or even dangerous and not necessary takeover situations [5]. Facial expressions can be analyzed by tracking of facial electromyographic activity, by manual coding of facial activity [6] as well as nowadays by automatic facial expression analysis using computer-vision algorithms [7]. Most of these techniques aim at identifying Action Units (AUs), which represents movements of an individual face muscle or muscle group [6]. Even though AUs do not interpret the meaning of expressions, relations of AU-pattern to basic emotions can be drawn [6]. In order to investigate discomfort-related changes of facial expressions / AUs, a driving simulator study has been carried out including standardized potentially uncomfortable situations during an automated trip. A total of four video cameras recorded the driver's face from different perspectives. These videos were analyzed with the Visage facial feature detection and face analysis software (Version 8.4, visagetechnologies.com), extracting values for 23 AUs for each video frame.

\section{Methods}

Driving Simulator Study Design. The study was conducted in a fixed-base driving simulator (Fig. 1A) with a $180^{\circ}$ horizontal field of view and fully equipped interior. A three minute trip was prerecorded by the investigators, containing three potentially uncomfortable situations. This trip was replayed while the participants sat in the driver's seat with no possibility to intervene by pedals or steering wheel. The situations consisted of three identical automated approaches to a truck driving ahead (Fig. 1B). The truck drove at a constant speed of $80 \mathrm{~km} / \mathrm{h}$, whereas the own car approached the truck in automated mode with $100 \mathrm{~km} / \mathrm{h}$. At a rather short distance of $9 \mathrm{~m}$, automated braking was initialized, reaching a minimum distance of $4.2 \mathrm{~m}$ (minimum time to contact of 1.1 s). A handset control was integrated into the driving simulator and the participants could report perceived discomfort continuously during the whole trip [8].

Participants. A total of 41 participants (24 male, 17 female) were recruited for the study, consisting of a younger age group under 40 years $(\mathrm{N}=20, \mathrm{M}=28$ years, $\mathrm{SD}=$ $3.9)$ and an older group over 60 years $(\mathrm{N}=21, \mathrm{M}=68$ years, $\mathrm{SD}=4.7)$. All participants gave written informed consent in accordance with the regulations and consent templates of the TU Chemnitz ethics commission. 


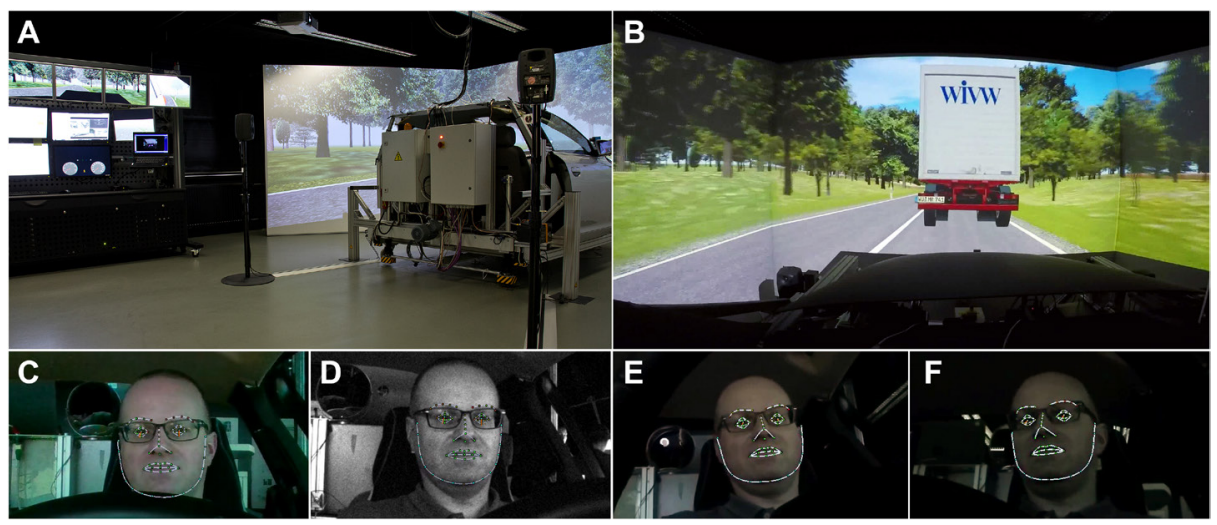

Fig. 1. Fixed-base driving simulator (A), approach situation to slower driving truck ahead (B), screenshots face tracking from video cameras Intel RealSense (C), AVT Mako (D), GoPro right side (E), GoPro left side (F). Written informed consent was obtained from the individual for the publication of this image.

Video Cameras and Face Tracking. The face of the driver was recorded by four video cameras to test face tracking with different cameras, different video formats and different perspectives (Fig. 1C-F). An Intel RealSense SR300 camera was placed exactly in front of the driver over the steering wheel (Fig. 1C), recoding a color video with a resolution of $1280 \times 720$ pixel and 30 frames per second (fps). An AVT Mako G-234B camera delivered a grayscale video of $640 \times 480$ pixel and $30 \mathrm{fps}$ (Fig. 1D). This camera was placed at the right side from the driver's perspective over the steering wheel. Two small GoPro Hero 5 video cameras were placed behind the steering wheel under the instrument cluster at the right (Fig. 1E) and left side (Fig. 1F) from the driver's perspective. Both cameras recorded a color video of 1920 x 1080 pixel with 50 fps. All video recordings were analyzed using the Visage facial feature detection and face analysis SDK (Version 8.4 for Windows, visagetechnologies.com). The SDK was integrated into an in-house developed data logging application, providing log files with 23 $\mathrm{AU}$ values as well as the tracking quality for each video frame.

\section{Results}

Discomfort Sequence Extraction. In order to assess AU-changes related to each of the three approach situations, each time period of pressing the handset control (independent of magnitude) was extracted as discomfort sequence. Having 41 participants and 3 situations, a theoretical sum of 123 sequences could be present. However, in 25 approach situations participants did not press the handset control at all. Thus, 98 sequences could be extracted with a mean duration of $7.1 \mathrm{~s}(\mathrm{SD}=4.5 \mathrm{~s})$. In addition, $10 \mathrm{~s}$ time intervals prior and after each discomfort sequence were extracted. As discomfort intervals varied in duration, a common percent time axis was created from $0 \%$ to $300 \%$ to show changes of each AU in one common scale (Fig. 2). Periods before and after the discomfort interval were always $10 \mathrm{~s}$ long; thus, $1 \%$ represents $0.1 \mathrm{~s}$. Each discomfort interval was 
divided into percent slices, and the mean of each AU was calculated over time of the respective percent slice. Further details on this method can be found in [8].

Raw Data Processing. Only video frames with an overall face tracking quality of $30 \%$ or more entered the analysis. To correct for high frequency signal fluctuations, a moving average over $\pm 2 \mathrm{~s}$ was calculated for each AU raw score. As raw AU-scores resulting from the face tracker are arbitrary decimal numbers, standardization is required to compare values between situations and participants. A common standardization procedure is the z-score, which expresses raw values as distance to the mean in units of standard deviations; with a total mean of zero and a standard deviation of one [8]. This transformation was applied for each sequence, resulting in relative changes within the $300 \%$ time period. Z-values were afterwards averaged over the 98 sequences (bold blue line in Fig. 2) and the $95 \%$ confidence interval was calculated pointwise and plotted as a light red area around the means. If the confidence band does not overlap between two particular points in time, these two means differ in a statistically significant manner.

Changes in AUs. Fig. 2 displays significant situation-related changes, which could be identified for 8 out of the 23 recorded AUs. To ensure stability and validity of results, only consistent findings over all four video cameras are reported. All charts in Fig. 2 belong to the Intel RealSense SR 300 camera video, however, the same trends were observed for the other three video cameras.
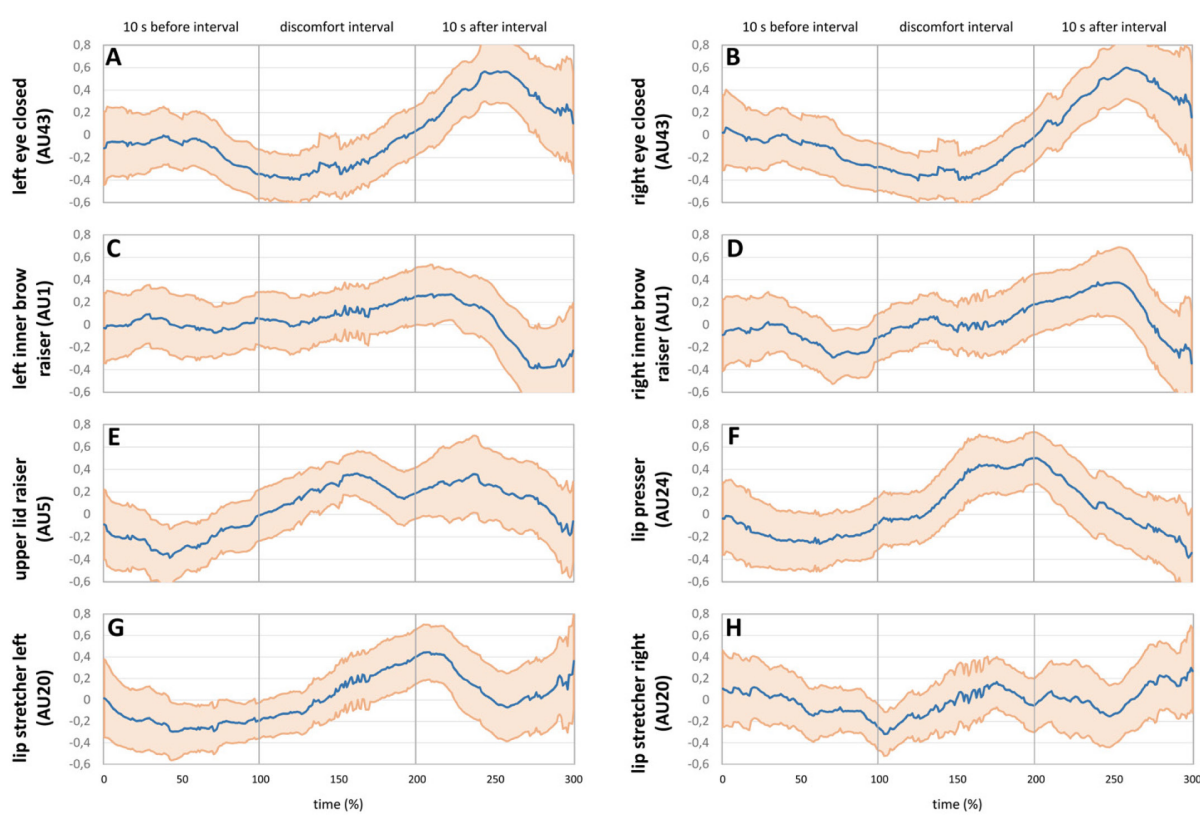

Fig. 2. Mean z-standardized AU values from the Intel RealSense SR 300 face video showing situation-related changes before, during and after reported discomfort intervals. The bold blue line shows the mean z-values over all participants and the three situations, the light red area shows the $95 \%$ pointwise confidence interval. 
During discomfort intervals, values of AU43 (eye closed) diminished significantly for the left eye (Fig. 2A) as well as for the right eye (Fig. 2B), with a rather steep increase towards the end and after the reported discomfort interval. The maximum was reached at about $5 \mathrm{~s}(250 \%)$ after the discomfort interval. This indicates continuous visual monitoring of the situation by keeping the eyes open and reducing eye blinks. A slightly increasing trend of AU1 (inner brow raiser) could be observed for the left (Fig. 2C) and right inner brow (Fig. 2D) until 5s (250\%) after the discomfort interval, with a sudden drop afterwards. The upper lid of both eyes (AU5, Fig. 2E) was raised during the discomfort interval, with a decreasing trend afterwards. A similar situation-related increase with subsequent decrease could be observed for AU24 (lip presser, Fig. 2F). The AU20 (lip stretcher) increased during the discomfort interval with a decrease afterwards; however the effect is more pronounced for the left lip side (Fig. 2G) than for the right side (Fig. 2H). No divergent or contradictory trends could be found for age groups or gender.

\section{$4 \quad$ Discussion and Conclusion}

The present study aimed at investigating discomfort-related changes of facial expressions using video-based automated analysis of facial AU. Facial expressions could be used to adapt automation features such as driving style aspects (e.g. speed and distance) to reduce discomfort and avoid non-necessary take-over situations [8].

In order to develop such a comfort-adaptive system, it is required to identify the potential of facial expressions as discomfort indicator, including relevance of AUs as well as magnitude, direction and timing of effects. Results showed that during discomfort intervals, both eye were kept open (AU43) and eye blinks were reduced, indicating continuous and attentive visual monitoring of the situation. This trend is in line with previous results based on eye-tracking, which showed reduced eye blink rate in this situation [8]. The raise of the inner brows (AU1) in association with the upper lid raiser (AU5) is considered as essential part in all prototypes and major variants of the emotion "surprise" [6]. The situation-related rises of lip pressing (AU24) as well as lip stretching (AU20) could be interpreted as sign for tension during the close approach situation. As these AU-trends could be observed in the face tracking results of all four video cameras from different perspectives, the findings could be regarded as rather stable for this situation. Additional analyses of individual and group differences showed of course different absolute rates of change in the AU raw scores per person. However, when standardizing these absolute values based on the individual trend (individual z-standardization), no divergent or contradictory trends could be found for subgroups such as age groups or gender.

To conclude, facial expressions captured by face tracking software showed specific changes for this uncomfortable approach situation in automated driving. Thus, facial expression analysis could contribute to adaptive automation, preventing human discomfort in the driver-vehicle team. Although the findings provide valuable indications of potentially relevant AUs as well as direction, magnitude and timing of effects, all analyses were carried out on aggregate level over all participants. Reliable and valid detection of discomfort on individual level requires further research on the stability and validity of these findings as well as the combination with additional driver state measures. 
Thus, further research steps in the MEDIATOR project are the validation of the findings in other potentially uncomfortable situations as well as the combination of face tracking data with additional driver state measures to finally develop and test a real-time capable mediating system.

\section{Funding}

Data collection was funded by the Federal Ministry of Education and Research under grant No. 16SV7690K (Project KomfoPilot). Data analysis of AUs was funded by the European Union's Horizon 2020 research and innovation programme under grant agreement No. 814735 (Project MEDIATOR).

\section{References}

1. ERTRAC: Connected Automated Driving Roadmap. European Road Transport Research Advisory Council, European Road Transport Research Advisory Council. https://www.ertrac.org/uploads/documentsearch/id57/ERTRAC-CAD-Roadmap-2019.pdf (2019)

2. Bellem, H., Thiel, B., Schrauf, M., Krems, J.F.: Comfort in automated driving: An analysis of preferences for different automated driving styles and their dependence on personality traits. Transportation Research Part F: Traffic Psychology and Behaviour 55, 90-100 (2018). doi: 10.1016/j.trf.2018.02.036

3. Elbanhawi, M., Simic, M., Jazar, R.: In the Passenger Seat: Investigating Ride Comfort Measures in Autonomous Cars. IEEE Intell. Transport. Syst. Mag. 7(3), 4-17 (2015). doi: 10.1109/MITS.2015.2405571

4. Beggiato, M., Hartwich, F., Schleinitz, K., Krems, J.F., Othersen, I., Petermann-Stock, I.: What would drivers like to know during automated driving? Information needs at different levels of automation., 7. Tagung Fahrerassistenz, Munich, 25.-26.11.2015. doi:10.13140/RG.2.1.2462.6007. (2015)

5. Techer, F., Ojeda, L., Barat, D., Marteau, J.-Y., Rampillon, F., Feron, S., Dogan, E.: Anger and highly automated driving in urban areas: The role of time pressure. Transportation Research Part F: Traffic Psychology and Behaviour 64, 353-360 (2019). doi: 10.1016/j.trf.2019.05.016

6. Ekman, P., Hager, J.C., Friesen, W.V.: Facial action coding system. The manual and investigator's Guide. Research Nexus, Salt Lake City (2002)

7. Ko, B.: A Brief Review of Facial Emotion Recognition Based on Visual Information. Sensors 18(2), 401 (2018). doi: 10.3390/s18020401

8. Beggiato, M., Hartwich, F., Krems, J.: Using Smartbands, Pupillometry and Body Motion to Detect Discomfort in Automated Driving. Front. Hum. Neurosci. 12, 3138 (2018). doi: 10.3389/fnhum.2018.00338 